\title{
Numerical Solution for Solving Fractional Differential Equations using Shifted Chebyshev Wavelet
}

\author{
Mohamed Elarabi Benattia * ${ }^{\text {I }}$ and Belghaba Kacem ${ }^{2}$ \\ 1,2 Laboratory of Mathematics and its Applications (LAMAP) \\ University of Oran 1, Ahmed Ben Bella \\ 1 mohamed.benattia74@yahoo.com ${ }^{2}$ belghaba@yahoo.fr
}

\begin{abstract}
In this paper, we are interested to develop a numerical method based on the Chebyshev wavelets for solving fractional order differential equations (FDEs). As a result of the presentation of Chebyshev wavelets, we highlight the operational matrix of the fractional order derivative through wavelet-polynomial matrix transformation which was utilized together with spectral and collocation methods to reduce the linear FDEs, to a system of algebraic equations. This method is a more simple technique of obtaining the operational matrix with straight forward applicability to the FDEs . The main characteristic behind the approach using this technique is that only a small number of shifted Chebyshev polynomials is needed to obtain a satisfactory results. Illustrative examples reveal that the present method is very effective and convenient for linear FDEs.
\end{abstract}

Keywords: Operational matrix, shifted Chebyshev wavelet, fractional derivatives, shifted Chebyshev polynomials, Caputo fractional derivative.

MSC2010 34A08.

\section{Introduction}

Over the last decades several analytical or approximate methods have been developed to solve fractional differential equations in many scientific areas. A particular attention has been given to solution studies of fractional ordinary differential equations, integral equations and fractional partial differential equations. Approximations and numerical methods are extensively used for the fractional differential equations do not have exact analytic solutions. The fractional derivative has generated mathematics tools perfectly adapted to different scientific fields. The fractional derivatives express very often the modeled and simulated properties (viscoelasticity for example) of different research domains such as fluid dynamics, heat and mass transfer, elasticity, etc [10, 13, 1, 16].

- The operational matrices of fractional order integration for Haar wavelets, Legendre wavelets, Chebyshev wavelets and Bernoulli wavelets have been developed in [2, 4, 5, 6, 15, respectively, to solve FDEs. A variant of theses methods, using shifted Legendre polynomial has been studied by [3]. It is natural to try to relate this work using described with shifted Chebyshev polynomials. Another motivation is the direct solution techniques for solving the derivatives forms of FDEs using shifted Chebyshev Tau method based on operational matrix of fractional derivative.

The outline of this paper is organized as follows. In section 1, we describe some basic definition and properties of fractional calculus. In section 2, we first defined shifted Chebyshev polynomial then, we describe some properties of Chebyshev wavelets. In Section 3, the shifted Chebyshev operational matrix of fractional order derivative and

\footnotetext{
${ }^{*}$ Corresponding author. Mohamed Elarabi Benattia ${ }^{1}$ mohamed.benattia74@yahoo.com
} 
Chebyshev wavelet operational matrix of fractional order derivative, in section 4 the proposed method is used to approximate solution of the problem. In section 5, application of the Chebyshev wavelets operational matrix of fractional order derivative. In section 6, the numerical examples of applying the method of this article are presented. Finally in section 7 , we achieve our work by some commentaries.

\section{Preliminaries}

\subsection{Fractional Derivative and Integral}

Here, we recall some basic definitions and properties The Riemann - Liouville integral $I$ of fractional order $\alpha$ of $f(t)$ is given by

$$
I^{\alpha} f(t)=\frac{1}{\Gamma(\alpha)} \int_{0}^{t}(t-s)^{\alpha-1} f(s) d s, \quad t>0, \alpha \geqslant 0
$$

its fractional derivative of order $\alpha>0$ is given by

$$
\left(D_{l}^{\alpha} f\right)(x)=\left(\frac{d}{d x}\right)^{m}\left(I^{m-\alpha} f\right)(x), \quad(\alpha>0, m-1<\alpha<m),
$$

where $\Gamma(:)$ is the gamma function which achieve the following properties:

$$
\left\{\begin{array}{c}
\Gamma(n+1)=n ! \\
\Gamma\left(n+\frac{1}{2}\right)=\frac{(2 n) !}{2^{2 n} n !} \sqrt{\pi} .
\end{array}, \forall n \in \mathbb{N} .\right.
$$

Some proprieties of $I^{\alpha}$ are as follows:

$$
\begin{gathered}
I^{\alpha} I^{\beta} f(t)=I^{\alpha+\beta} f(t), \quad \alpha>0, \quad \beta>0 . \\
I^{\alpha} t^{\beta}=\frac{\Gamma(\beta+1)}{\Gamma(\beta+\alpha+1)} t^{\beta+\alpha} .
\end{gathered}
$$

The Caputo fractional derivative $D^{\alpha}$ of a function $f(t)$ is defined as

$$
D^{\alpha} f(t)=\frac{1}{\Gamma(n-\alpha)} \int_{0}^{t} \frac{f^{(n)}(s)}{(t-s)^{\alpha-n+1}} d s, \quad n-1<\alpha \leqslant n, n \in \mathbb{N} .
$$

The following are some proprieties of Caputo fractional derivatives

$$
\begin{gathered}
D^{\alpha} C=0, \quad(C \text { is constant }), \\
D^{\alpha} t^{\beta}=\left\{\begin{array}{c}
0, \beta \in \mathbb{N} \cup\{0\} \quad \text { and } \beta<\lceil\alpha\rceil \\
\frac{\Gamma(\beta+1)}{\Gamma(\beta-\alpha+1)} t^{\beta-\alpha}, \beta \in \mathbb{N} \cup\{0\} \quad \text { and } \beta \geqslant\lceil\alpha\rceil \quad \text { or } \beta \notin \mathbb{N} \text {. and } \beta>\lfloor\alpha\rfloor,
\end{array}\right.
\end{gathered}
$$

where $\lceil\alpha\rceil$ denote the smallest integer greater than or equal to $\alpha$ and $\lfloor\alpha\rfloor$ denote the largest integer less than or equal to $\alpha$.

The Caputo fractional deferential operator is a linear operator, since,

$$
D^{\alpha}(\lambda f(t)+\mu g(t))=\lambda D^{\alpha} f(t)+\mu D^{\alpha} g(t)
$$

where $\lambda$ and $\mu$ are constants. 


\section{Chebyshev Polynomial and Chebyshev Wavelets}

\subsection{Properties of shifted Chebyshev polynomials}

The well known Chebyshev polynomials of degree $m$ are defined on the interval $[-1,1]$ and can be determined with the aid of the following recurrence formula

$$
T_{m+1}(t)=2 t T_{m}(t)-T_{m-1}(t), \quad m=1,2, . .,
$$

where $T_{0}(t)=1$ and $T_{1}(t)=t$. For one to use these polynomials on the interval [0,1], we define the so called shifted Chebyshev polynomials by using the change of variable $t=2 x-1$. Let the shifted Chebyshev polynomials $T_{m}(2 x-1)$ be denoted by $T_{m}^{\star}(x)$ Then $T_{m}^{\star}(x)$ can be obtained as follows:

$$
T_{m+1}^{\star}(x)=2(2 x-1) T_{m}^{\star}(x)-T_{m-1}^{\star}(x), \quad m=1,2, . .,
$$

where $T_{0}^{\star}(x)=1, T_{1}^{\star}(x)=2 x-1$ and $T_{2}^{\star}(x)=2(2 x-1)^{2}-1=8 x^{2}-8 x+1$.

The analytic form of the shifted Chebyshev polynomials $T_{m}^{\star}(x)$ of degree $m$ is given by

$$
T_{m}^{\star}(x)=m \sum_{k=0}^{m}(-1)^{m-k} \frac{(m+k-1) ! 2^{2 k}}{(m-k) !(2 k) !} x^{k} .
$$

The orthogonality condition is

$$
\int_{0}^{1} \frac{T_{m}^{\star}(x) T_{n}^{\star}(x)}{\sqrt{1-(2 x-1)^{2}}}=\left\{\begin{array}{cc}
\frac{\pi \gamma_{m}}{4}, & m=n \\
0, & m \neq n
\end{array}\right.
$$

where

$$
\gamma_{m}= \begin{cases}2, & m=0 \\ 1, & m \geqslant 1\end{cases}
$$

\subsection{Wavelets and Chebyshev Wavelets}

In recent years, wavelets have found their way into many different fields of science and engineering. Wavelets constitute a family of functions constructed from dilation and translation of single function called the mother wavelet. When the dilation parameter $a$ and the translation parameter $b$ vary continuously, we have the following family of continuous wavelets:

$$
\psi_{a, b}(t)=|a|^{-\frac{1}{2}} \psi\left(\frac{t-b}{a}\right) \quad a, b \in \mathbb{R}, a \neq 0 .
$$

Chebyshev wavelets $\psi_{n m}(t)=\psi(k, n, m, t)$ have four arguments; $n$ argument $k$ can assume any positive integer, $m$ is the order for Chebyshev polynomials and $t$ is the normalized time. They are defined on the interval $[0,1)$ by

$$
\psi_{n, m}(t)=\left\{\begin{array}{cc}
2^{\frac{k+1}{2}} P_{m}\left(2^{k+1} t-2 n-1\right), & \frac{n}{2^{k}} \leqslant t<\frac{n+1}{2^{k}} \\
0, & \text { otherwise }
\end{array},\right.
$$

where

$$
P_{m}(t)=\left\{\begin{array}{cl}
\frac{1}{\sqrt{\pi}} & , m=0 . \\
\sqrt{\frac{2}{\pi}} T_{m}(t) & , m \geqslant 1 .
\end{array}\right.
$$

\subsection{Function Approximations}

A function $f(t)$ defined over $L^{2}[0,1]$ can be expanded in the terms of Chebyshev wavelets as

$$
f(t)=\sum_{n=0}^{\infty} \sum_{m=0}^{\infty} c_{n m} \psi_{n m}(t)
$$

where the coefficient $c_{n, m}$ is given by

$$
c_{n, m}=\left\langle f(t), \psi_{n, m}(t)\right\rangle,
$$


in which $\langle.,$.$\rangle denotes the inner product. If the infinite series in \sqrt{13}$ is truncated, then it can be written as

$$
f(t)=\sum_{n=0}^{2^{k}-1} \sum_{m=0}^{M} c_{n m} \psi_{n m}(t)=C^{T} \Psi(t)
$$

Where $C$ and $\Psi(t)$ are $2^{k}(M+1) \times 1$ matrices given by

$$
\left\{\begin{array}{c}
C=\left[c_{0,0}, c_{0,1}, \ldots, c_{0, M}, c_{1,0} c_{1,1}, \ldots c_{1, M}, \ldots ., c_{\left(2^{k}-1\right), 0}, c_{\left(2^{k}-1\right), 1}, \ldots ., c_{\left(2^{k}-1\right), M}\right] \\
\Psi=\left[\psi_{0,0}, \psi_{0,1}, \ldots, \psi_{0, M}, \psi_{1,0}, \psi_{1,1}, \ldots \psi_{1, M}, \ldots ., \psi_{\left(2^{k}-1\right), 0}, \psi_{\left(2^{k}-1\right), 1}, \ldots ., \psi_{\left(2^{k}-1\right), M}\right]^{T} .
\end{array}\right.
$$

\section{Chebyshev Wavelet Operational Matrix of Fractional Order Deriva- tive}

In this section, we drive the Chebyshev wavelet operational matrix of fractional derivative by first transforming the wavelets to shifted Chebyshev polynomials, we then make use of the shifted Chebyshev operational matrix of the fractional derivative in, and finally we derive the Chebyshev wavelet operational matrix of the fractional derivative.

\subsection{Transformation Matrix of the Chebyshev Wavelets to Chebyshev polynomials}

An arbitrary function $y(t) \in L^{2}[0,1]$ can be expanded into shifted Chebyshev polynomials as

$$
y(x)=\sum_{m=0}^{M} p_{m} T_{m}^{\star}(x)=P \Psi(x),
$$

where the shifted Chebyshev coefficient vector $P$ and the shifted Chebyshev vector $\Psi^{\prime}(x)$ are given by

$$
\begin{gathered}
P=\left[p_{0}, p_{1}, \ldots \ldots, p_{M}\right], \\
\Psi^{\prime}(x)=\left[T_{0}^{\star}(x), T_{1}^{\star}(x), \ldots, T_{m}^{\star}(x)\right] .
\end{gathered}
$$

The Chebyshev wavelet may be expanded in to $(M+1)$ terms shifted Chebyshev polynomials as

$$
\Psi_{2^{k}(M+1) \times 1}(t)=\Phi_{2^{k}(M+1) \times(M+1)} \Psi_{(M+1) \times 1}^{\prime},
$$

where $\Phi$ is the transformation matrix of the Chebyshev wavelet to Chebyshev polynomials. Let $M=2$ and $k=1$, we have

$$
\left\{\begin{array}{c}
\Psi=\left[\psi_{0,0}, \psi_{0,1}, \psi_{0,2}, \psi_{1,0} \psi_{1,1}, \psi_{1,2}\right]^{T} \\
\Psi^{\prime}(x)=\left[T_{0}^{\star}(x), T_{1}^{\star}(x), T_{2}^{\star}(x)\right]
\end{array},\right.
$$

where

$$
\left\{\begin{array}{c}
\psi_{0,0}=2 P_{0}(4 t-1)=\frac{2}{\sqrt{\pi}} T_{0}^{\star}(t) \\
\psi_{0,1}=2 P_{1}(4 t-1)=2 \sqrt{\frac{2}{\pi}} T_{1}(4 t-1)=2 \sqrt{\frac{2}{\pi}}(4 t-1)=2 \sqrt{\frac{2}{\pi}}(2(2 t-1)+1) \\
=4 \sqrt{\frac{2}{\pi}} T_{1}^{\star}(t)+2 \sqrt{\frac{2}{\pi}} T_{0}^{\star}(t) \\
\psi_{0,2}=2 P_{2}(4 t-1)=2 \sqrt{\frac{2}{\pi}} T_{2}(4 t-1)=2 \sqrt{\frac{2}{\pi}}\left[2(4 t-1) T_{1}(4 t-1)-1\right] \quad, \quad 0 \leqslant t<\frac{1}{2} \\
=2 \sqrt{\frac{2}{\pi}}[2(4 t-1)(4 t-1)-1]=2 \sqrt{\frac{2}{\pi}}\left[8(2 t-1)^{2}+8(2 t-1)+1\right] \\
=8 \sqrt{\frac{2}{\pi}} T_{2}^{\star}(t)+16 \sqrt{\frac{2}{\pi}} T_{1}^{\star}(t)+10 \sqrt{\frac{2}{\pi}} T_{0}^{\star}(t)
\end{array}\right.
$$




$$
\left\{\begin{array}{c}
\psi_{1,0}=2 P_{0}(4 t-3)=\frac{2}{\sqrt{\pi}} T_{0}^{\star}(t) \\
\psi_{1,1}=2 P_{1}(4 t-3)=2 \sqrt{\frac{2}{\pi}} T_{1}(4 t-3)=2 \sqrt{\frac{2}{\pi}}(4 t-3)=2 \sqrt{\frac{2}{\pi}}(2(2 t-1)-1) \\
=4 \sqrt{\frac{2}{\pi}} T_{1}^{\star}(t)-2 \sqrt{\frac{2}{\pi}} T_{0}^{\star}(t) \\
\psi_{1,2}=2 P_{2}(4 t-3)=2 \sqrt{\frac{2}{\pi}} T_{2}(4 t-3)=2 \sqrt{\frac{2}{\pi}}\left[2(4 t-3) T_{1}(4 t-3)-1\right] \quad, \quad \frac{1}{2} \leqslant t<1 \\
=2 \sqrt{\frac{2}{\pi}}[2(4 t-3)(4 t-3)-1]=2 \sqrt{\frac{2}{\pi}}\left[8(2 t-1)^{2}-8(2 t-1)+1\right] \\
=8 \sqrt{\frac{2}{\pi}} T_{2}^{\star}(t)-16 \sqrt{\frac{2}{\pi}} T_{1}^{\star}(t)+10 \sqrt{\frac{2}{\pi}} T_{0}^{\star}(t)
\end{array}\right.
$$

Thus, in this case

$$
\Phi= \begin{cases}\Phi_{1}=\left[a_{i, j}\right] 6 \times 3, & 0 \leqslant t<\frac{1}{2} \\ \Phi_{2}=\left[b_{i, j}\right] 6 \times 3, & \frac{1}{2} \leqslant t<1\end{cases}
$$

where

$$
\Phi_{1}=\left(\begin{array}{ccc}
\frac{2}{\sqrt{\pi}} & 0 & 0 \\
2 \sqrt{\frac{2}{\pi}} & 4 \sqrt{\frac{2}{\pi}} & 0 \\
10 \sqrt{\frac{2}{\pi}} & 16 \sqrt{\frac{2}{\pi}} & 8 \sqrt{\frac{2}{\pi}} \\
0 & 0 & 0 \\
0 & 0 & 0 \\
0 & 0 & 0
\end{array}\right), \quad \Phi_{2}=\left(\begin{array}{ccc}
0 & 0 & 0 \\
0 & 0 & 0 \\
0 & 0 & 0 \\
\frac{2}{\sqrt{\pi}} & 0 & 0 \\
-2 \sqrt{\frac{2}{\pi}} & 4 \sqrt{\frac{2}{\pi}} & 0 \\
10 \sqrt{\frac{2}{\pi}} & -16 \sqrt{\frac{2}{\pi}} & 8 \sqrt{\frac{2}{\pi}}
\end{array}\right) .
$$

\subsection{Chebyshev operational matrix to fractional calculus}

The fractional derivative of order $\alpha$ of the vector $\Psi^{\prime}(t)$ can be expressed by

$$
D^{\alpha} \Psi^{\prime}(t)=L^{(\alpha)} \Psi^{\prime}(t)
$$

where $L^{(\alpha)}$ is the $(m+1) \times(m+1)$ operational matrix of fractional derivative of order $\alpha$.

In the following theorem we generalize the operational matrix of derivative of shifted Chebyshev polynomials for fractional derivative. The $L^{(\alpha)}$ operational matrix of fractional derivative of order $\alpha$ defined as:

$$
L^{(\alpha)}=\left(\begin{array}{cccc}
0 & 0 & \cdots & 0 \\
\vdots & \vdots & \cdots & \vdots \\
0 & 0 & \cdots & 0 \\
\sum_{k=\lceil\alpha\rceil}^{\lceil\alpha\rceil} \vartheta_{\lceil\alpha\rceil, 0, k} & \sum_{k=\lceil\alpha\rceil}^{\lceil\alpha\rceil} \vartheta_{\lceil\alpha\rceil, 1, k} & \cdots & \sum_{k=\lceil\alpha\rceil}^{\lceil\alpha\rceil} \vartheta_{\lceil\alpha\rceil, m, k} \\
\vdots & \vdots & \cdots & \vdots \\
\sum_{k=\lceil\alpha\rceil}^{i} \vartheta_{i, 0, k} & \sum_{k=\lceil\alpha\rceil}^{i} \vartheta_{i, 1, k} & \cdots & \sum_{k=\lceil\alpha\rceil}^{i} \vartheta_{i, m, k} \\
\vdots & \vdots & \ldots & \vdots \\
\sum_{k=\lceil\alpha\rceil}^{m} \vartheta_{m, 0, k} & \sum_{k=\lceil\alpha\rceil}^{m} \vartheta_{m, 1, k} & \cdots & \sum_{k=\lceil\alpha\rceil}^{m} \vartheta_{m, m, k}
\end{array}\right)
$$

where $\vartheta_{i, j, k}$ is given by:

$$
\left\{\begin{array}{c}
\vartheta_{i, j, k}=\frac{4 i j}{\pi} \sum_{l=0}^{j}(-1)^{i+j-k-l} \frac{(j+l-1) !(i+k-1) ! k ! 2^{2 l+2 k}}{(j-l) !(i-k) !(2 l) !(2 k) !(k+l-\alpha+1) \Gamma(k-\alpha+1)}, \quad j \geqslant 1 . \\
\vartheta_{i, j, k}=(-1)^{i-k} \frac{2 i}{\pi(k-\alpha+1)} \frac{(i+k-1) ! 2^{2 k} k !}{(i-k) !(2 k) ! \Gamma(k-\alpha+1)}, \quad j=0 .
\end{array}\right.
$$


Proof. The analytic form of the shifted Chebyshev polynomial $T_{i}^{\star}(t)$ of degree $i$ given by

$$
T_{i}^{\star}(x)=i \sum_{k=0}^{i}(-1)^{i-k} \frac{(i+k-1) ! 2^{2 k}}{(i-k) !(2 k) !} x^{k} .
$$

Note that $T_{i}^{\star}(0)=(-1)^{i}$ and $T_{i}^{\star}(1)=1$. Using equations (6), (7) and 21] we have

$$
\left\{\begin{array}{c}
D^{\alpha} T_{i}^{\star}(x)=i \sum_{k=0}^{i}(-1)^{i-k} \frac{(i+k-1) ! 2^{2 k}}{(i-k) !(2 k) !} D^{\alpha}\left(x^{k}\right) \\
=i \sum_{k=\lceil\alpha\rceil}^{i}(-1)^{i-k} \frac{(i+k-1) ! 2^{2 k} k !}{(i-k) !(2 k) ! \Gamma(k-\alpha+1)} x^{k-\alpha} \quad, \quad i=\lceil\alpha\rceil, \ldots, m .
\end{array}\right.
$$

Now, approximate $x^{k-\alpha}$ by $(m+1)$ terms of shifted Chebyshev series, we have

$$
x^{k-\alpha} \approx \sum_{j=0}^{m} b_{k j} T_{j}^{\star}(x)
$$

where

$$
b_{k 0}=\frac{2}{\pi} \int_{0}^{1} x^{k-\alpha} T_{0}^{\star}(x) d x=\frac{2}{\pi} \int_{0}^{1} x^{k-\alpha}=\frac{2}{\pi(k-\alpha+1)},
$$

and

so

$$
\left\{\begin{aligned}
& b_{k, j}=\frac{4}{\pi} \int_{0}^{1} x^{k-\alpha} T_{j}^{\star}(x) d x \\
= & \frac{4}{\pi} \int_{0}^{1} x^{k-\alpha} j \sum_{l=0}^{j}(-1)^{j-l} \frac{(j+l-1) ! 2^{2 l}}{(j-l) !(2 l) !} x^{l} d x \quad, \quad j \geqslant 1, \\
= & \frac{4 j}{\pi} \int_{0}^{1} \sum_{l=0}^{j}(-1)^{j-l} \frac{(j+l-1) ! 2^{2 l}}{(j-l) !(2 l) !} x^{k-\alpha+l} d x \\
= & \frac{4 j}{\pi} \sum_{l=0}^{j}(-1)^{j-l} \frac{(j+l-1) ! 2^{2 l}}{(j-l) !(2 l) !(k-\alpha+l+1)},
\end{aligned}\right.
$$

$$
b_{k, j}=\frac{4 j}{\pi} \sum_{l=0}^{j}(-1)^{j-l} \frac{(j+l-1) ! 2^{2 l}}{(j-l) !(2 l) !(k-\alpha+l+1)}, \quad j \geqslant 1 .
$$

Employing the equations 22 and 25 , we get

$$
\left\{\begin{aligned}
D^{\alpha} T_{i}^{\star}(x) & \simeq i \sum_{k=\lceil\alpha\rceil}^{i}(-1)^{i-k} \frac{(i+k-1) ! 2^{2 k} k !}{(i-k) !(2 k) ! \Gamma(k-\alpha+1)} \sum_{j=0}^{m} b_{k j} T_{j}^{\star}(x) . \\
& \simeq i \sum_{k=\lceil\alpha\rceil}^{i}(-1)^{i-k} \frac{(i+k-1) ! 2^{2 k} k !}{(i-k) !(2 k) ! \Gamma(k-\alpha+1)} \sum_{j=0}^{m} b_{k j} T_{j}^{\star}(x) \\
& \simeq i \sum_{k=\lceil\alpha\rceil j=0}^{i} \sum_{j=0}^{m}(-1)^{i-k} \frac{(i+k-1) ! 2^{2 k} k !}{(i-k) !(2 k) ! \Gamma(k-\alpha+1)} b_{k j} T_{j}^{\star}(x) \\
& =\sum_{j=0}^{m}\left(\sum_{k=\lceil\alpha\rceil}^{i} \vartheta_{i, j, k}\right) T_{j}^{\star}(x)
\end{aligned}\right.
$$

where

$$
\vartheta_{i, j, k}=\frac{4 i j}{\pi} \sum_{l=0}^{j}(-1)^{i+j-k-l} \frac{(j+l-1) !(i+k-1) ! k ! 2^{2 l+2 k}}{(j-l) !(i-k) !(2 l) !(2 k) !(k+l-\alpha+1) \Gamma(k-\alpha+1)}, i=\lceil\alpha\rceil, . ., m, j \geqslant 1 .
$$

Employing the equations 24) and 25), we get

$$
\left\{\begin{array}{l}
\vartheta_{i, 0, k}=i(-1)^{i-k} \frac{(i+k-1) ! 2^{2 k} k !}{(i-k) !(2 k) ! \Gamma(k-\alpha+1)} b_{k, 0} \\
=(-1)^{i-k} \frac{2 i}{\pi(k-\alpha+1)} \frac{(i+k-1) ! 2^{2 k} k !}{(i-k) !(2 k) ! \Gamma(k-\alpha+1)}
\end{array}\right.
$$


Where $\vartheta_{i, j, k}$ is given in equation 20 . Rewrite equation 26 as a vector form we have

$$
D^{\alpha} T_{i}^{\star}(x)=\left(\sum_{k=\lceil\alpha\rceil}^{i} \vartheta_{i, 0, k}, \sum_{k=\lceil\alpha\rceil}^{i} \vartheta_{i, 1, k}, \ldots \ldots ., \sum_{k=\lceil\alpha\rceil}^{i} \vartheta_{i, m, k}\right) \Psi^{\prime}(x), \quad i=\lceil\alpha\rceil, . ., m .
$$

\subsection{Chebyshev Wavelet Operational Matrix of Fractional Order Derivative}

Now, we derive Chebyshev wavelet operational matrix of fractional order derivative. Let

$$
D^{\alpha} \Psi(t)=H^{(\alpha)} \Psi(t),
$$

where $H^{(\alpha)}$ is the Chebyshev wavelet operational matrix of fractional order derivative. Using (18) and (19) we get

$$
D^{\alpha} \Psi(t)=D^{\alpha} \Phi \Psi^{\prime}(t)=\Phi D^{\alpha} \Psi^{\prime}(t)=\Phi L^{(\alpha)} \Psi^{\prime}(t),
$$

from equation $(30)$ and $(31)$, we have

$$
H^{(\alpha)} \Psi(t)=H^{(\alpha)} \Phi \Psi^{\prime}(t)=\Phi L^{(\alpha)} \Psi^{\prime}(t) .
$$

Thus, the Chebyshev wavelet operational matrix of fractional derivative $H^{(\alpha)}$ is given by

$$
H^{(\alpha)}=\Phi L^{(\alpha)} \Phi^{-1}
$$

\section{Applications of the operational matrix of fractional derivative}

In this section, in order to show the high importance of operational matrix of fractional derivative, we apply it to solve multi-order fractional differential equation.

\subsection{Linear multi-order fractional differential equation}

Consider the linear multi-order fractional differential equation

$$
D^{\alpha} y(x)=a_{1} D^{\mu_{1}} y(x)+a_{2} D^{\mu_{2}} y(x)+\ldots+a_{s} D^{\mu_{s}} y(x)+a_{s+1} y(x)+a_{s+2} g(x),
$$

with initial conditions

$$
y^{(i)}(x)=d_{i}, i=1, . ., n .
$$

Where $a_{j}$, for $j=1, \ldots \ldots, s+2$ are real constant coefficients and also $n<\alpha \leqslant n+1$, $0<\mu_{1}<\mu_{2}<\ldots .<\mu_{s}<\alpha . D^{\alpha}$ denotes the Caputo fractional derivative of order $\alpha$.

To solve the problem (34) and (35), we approximate $y(x)$ and $g(x)$ by the Chebyshev wavelets as,

$$
\begin{aligned}
& y(x) \approx \sum_{n=0}^{2^{k}-1} \sum_{m=0}^{M} c_{n, m} \psi_{n, m}=C^{T} \Psi(t) . \\
& g(x) \approx \sum_{n=0}^{2^{k}-1} \sum_{m=0}^{M} z_{n, m} \psi_{n, m}=G^{T} \Psi(t),
\end{aligned}
$$

where

$$
G=\left[z_{0,0}, z_{0,1}, \ldots, z_{0, M}, z_{1,0}, z_{1,1}, \ldots z_{1, M}, \ldots, z_{\left(2^{k}-1\right), 0}, z_{\left(2^{k}-1\right), 1}, \ldots, z_{\left(2^{k}-1\right), M}\right],
$$

is know but $C$ as defined in 15 is the unknown vector.

Now, using $(30)$ and $(36)$ we get

$$
\begin{aligned}
D^{\alpha} y(x) & \approx C^{T} D^{\alpha} \Psi(x) \approx C^{T} H^{(\alpha)} \Psi(x) \\
D^{\mu_{j}} y(x) & \approx C^{T} D^{\mu_{j}} \Psi(x) \approx C^{T} H^{\left(\mu_{j}\right)} \Psi(x) .
\end{aligned}
$$


Using (36), 39) the residual $R(x)$ for equation (34) can be written as

$$
R(x) \approx\left(C^{T} H^{(\alpha)} \Psi(x)-a_{1} C^{T} H^{\left(\mu_{1}\right)} \Psi(x)-\ldots . a_{k} C^{T} H^{\left(\mu_{k}\right)} \Psi(x)-a_{k+1} C^{T} \Psi(x)-a_{k+2} G^{T} \Psi(x)\right) .
$$

As in typical tau method we generate $2^{k}(M+1)-n$ linear equations by applying

$$
\langle R(x), \Psi(x)\rangle=\int_{0}^{1} R(x) \Psi(x) d x, \quad j=1, \ldots, 2^{k}(M+1)-n .
$$

Also by substituting initial conditions 35 in to 36 and 39 we have

$$
\left\{\begin{array}{c}
y(0) \approx C^{T} \Psi(0)=d_{0} \\
y^{\prime}(0) \approx C^{T} H^{(1)} \Psi(0)=d_{1} \\
\vdots \\
y^{(n)}(0) \approx C^{T} H^{(n)} \Psi(0)=d_{n}
\end{array}\right.
$$

Equations 41 and 42 generate $2^{k}(M+1)$ set of linear equations. These linear equations can be solved for unknown coefficients of the vector $C$.

\section{Illustrative Examples}

In this section, we demonstrate the effectiveness of the proposed Chebyshev wavelet method with numerical examples. we consider the following initial value problem,

$$
D^{2} y(x)+D^{\frac{1}{2}} y(x)+y(x)=g(x)
$$

with $g(x)=x^{2}+2+\frac{8}{3 \sqrt{\pi}} x^{\frac{3}{2}}$.

The exact solution of this problem is $y(x)=x^{2}$. By applying the technique described in the section (??) with $M=2$ and $k=0$ we may write the approximate solution as

$$
\left\{\begin{array}{c}
y(x)=C^{T} \Psi(x) \Longleftrightarrow y(0)=C^{T} \Psi(0)=d_{0}=0 . \\
y^{\prime}(x)=C^{T} H^{(1)} \Psi(x) \Longleftrightarrow y^{\prime}(0)=C^{T} H^{(1)} \Psi(0)=d_{1}=0 .
\end{array}\right.
$$

Now, we calculate $\Psi(x)=\left(\psi_{00}(x), \quad \psi_{01}(x), \quad \psi_{02}(x)\right)^{T}$, where

$$
\left\{\begin{array}{c}
\psi_{00}(x)=\sqrt{2} P_{0}(x)=\sqrt{\frac{2}{\pi}} \\
\psi_{01}(x)=\sqrt{2} P_{1}(2 x-1)=\sqrt{2} \sqrt{\frac{2}{\pi}} T_{1}(2 x-1)=\frac{2}{\sqrt{\pi}}(2 x-1) \\
\psi_{01}(x)=\sqrt{2} P_{2}(2 x-1)=\sqrt{2} \sqrt{\frac{2}{\pi}} T_{2}(2 x-1)=\frac{2}{\sqrt{\pi}}\left[2(2 x-1)^{2}-1\right]=\frac{2}{\sqrt{\pi}}\left(8 x^{2}-8 x+1\right)
\end{array} .\right.
$$

So

$$
\begin{gathered}
\Psi(x)=\left(\begin{array}{c}
\sqrt{\frac{2}{\pi}} \\
\frac{2}{\sqrt{\pi}}(2 x-1) \\
\frac{2}{\sqrt{\pi}}\left(8 x^{2}-8 x+1\right)
\end{array}\right) \Longrightarrow \Psi(0)=\left(\begin{array}{c}
\sqrt{\frac{2}{\pi}} \\
-\frac{2}{\sqrt{\pi}} \\
\frac{2}{\sqrt{\pi}}
\end{array}\right) . \\
y(0)=C^{T} \Psi(0)=d_{0}=0 \Longleftrightarrow\left(c_{00}, c_{01}, c_{02}\right)\left(\begin{array}{c}
\sqrt{\frac{2}{\pi}} \\
-\frac{2}{\sqrt{\pi}} \\
\frac{2}{\sqrt{\pi}}
\end{array}\right)=0 \text { which implies } \\
c_{00}-\sqrt{2} c_{01}+\sqrt{2} c_{02}=0 .
\end{gathered}
$$


Now, we calculate the matrices $L^{(1)}, L^{(2)}$ and $L^{\left(\frac{1}{2}\right)}$. From the equation 20 we have

$$
L^{(1)}=\left(\begin{array}{ccc}
0 & 0 & 0 \\
\frac{4}{\pi} & 0 & \frac{-8}{3 \pi} \\
0 & \frac{16}{3 \pi} & 0
\end{array}\right), \quad L^{(2)}=\left(\begin{array}{ccc}
0 & 0 & 0 \\
0 & 0 & 0 \\
\frac{32}{\pi} & 0 & \frac{-64}{3 \pi}
\end{array}\right), \quad L^{\left(\frac{1}{2}\right)}=\left(\begin{array}{ccc}
0 & 0 & 0 \\
\frac{4}{3 \pi \sqrt{\pi}} & \frac{24}{5 \pi \sqrt{\pi}} & \frac{-24}{7 \pi \sqrt{\pi}} \\
\frac{-4}{15 \pi \sqrt{\pi}} & \frac{24}{7 \pi \sqrt{\pi}} & \frac{824}{135 \pi}
\end{array}\right),
$$

using the equation 33 and

$$
\Phi=\left(\begin{array}{ccc}
\sqrt{\frac{2}{\pi}} & 0 & 0 \\
0 & \frac{2}{\sqrt{\pi}} & 0 \\
0 & 0 & \frac{2}{\sqrt{\pi}}
\end{array}\right)
$$

we get the matrices $H^{(1)}, H^{(2)}$ and $H^{\left(\frac{1}{2}\right)}$ as follow:

$$
H^{(1)}=\left(\begin{array}{ccc}
0 & 0 & 0 \\
\frac{4 \sqrt{2}}{\pi} & 0 & \frac{-8}{3 \pi} \\
0 & \frac{16}{3 \pi} & 0
\end{array}\right), H^{(2)}=\left(\begin{array}{ccc}
0 & 0 & 0 \\
0 & 0 & 0 \\
\frac{32 \sqrt{2}}{\pi} & 0 & \frac{-64}{3 \pi}
\end{array}\right), H^{\left(\frac{1}{2}\right)}=\left(\begin{array}{ccc}
0 & 0 & 0 \\
\frac{4 \sqrt{2 \pi}}{3 \pi} & \frac{24 \sqrt{\pi}}{5 \pi} & \frac{-24 \sqrt{\pi}}{7 \pi} \\
\frac{-4 \sqrt{2 \pi}}{15 \pi} & \frac{24 \sqrt{\pi}}{7 \pi} & \frac{824 \sqrt{\pi}}{135 \pi}
\end{array}\right) .
$$

Then, applying the equations 42 we obtain

$$
c_{01}-4 c_{02}=0
$$

We can expand the function $g(x)$ of the problem by Chebyshev wavelets as

$$
g(x)=\left(\begin{array}{lll}
2.513562154 & 1.1221745532 & 0.2077870929
\end{array}\right) \Psi(x) .
$$

And by using the equation 41, we get

$$
\frac{4 \sqrt{2 \pi}}{3 \pi} c_{01}-\frac{4 \sqrt{2 \pi}}{15 \pi} c_{02}+\frac{32 \sqrt{2}}{\pi} c_{02}+c_{00}=2.513562154 .
$$

Solving the following system by the Gauss elimination

$$
\left\{\begin{array}{c}
c_{00}-\sqrt{2} c_{01}+\sqrt{2} c_{02}=0 \\
c_{01}-4 c_{02}=0 \\
\frac{4 \sqrt{2 \pi}}{3 \pi} c_{01}-\frac{4 \sqrt{2 \pi}}{15 \pi} c_{02}+\frac{32 \sqrt{2}}{\pi} c_{02}+c_{00}=2.513562154
\end{array},\right.
$$

we obtain

$$
c_{00}=0.4699864272, \quad c_{01}=0.4431074542, \quad c_{02}=0.1107768636 .
$$

Finally,

$$
y(x)=\left(\begin{array}{lll}
0.4699864272 & 0.4431074542 & 0.1107768636
\end{array}\right)\left(\begin{array}{c}
\sqrt{\frac{2}{\pi}} \\
\frac{2}{\sqrt{\pi}}(2 x-1) \\
\frac{2}{\sqrt{\pi}}\left(8 x^{2}-8 x+1\right)
\end{array}\right) \approx x^{2}
$$

\section{Conclusion}

In this article, a general formulation foe deriving the Chebyshev wavelet operational matrix of fractional derivatives has been derived, and as an important application, we describe how to solve numerically the FDEs. Maple software is used to obtain the approximate solution. 


\section{References}

[1] A. Hanyga, Fractional-order relaxation laws in non-linear viscoelasticity, Continuum Mechanics and Thermodynamics, 19 (2007), 25-36.

[2] A. H. Bhrawy, A. S. Alofi. The operational matrix of fractional integration for shifted Chebyshev polynomials. AML 26(2013)25-31.

[3] A. Isah, P. Chang. Legendre Wavelet Operational Matrix of Fractional Derivative Through Wavelet-Polynomial Transformation and its Application in Solving Fractional Order Differential Equations. IJPAM. 105(2015), 97114.

[4] A. Saadatmandia, M. Dehghanb. Anew operational matrix for solving fractional-order differential equations.CMA 59(2010) 1326-1336.

[5] E. Keshavarz, Y. Ordokhani, M. Razzaghi. Bernoulli wavelet operational matrix of fractional order integration and its applications in solving the fractional order differential equations. Applied Mathematical Modelling 38 (2014) 6038-6051.

[6] F. Mohammadi. Numerical solution of Bagley-Torvik equation using Chebyshev wavelet operational matrix of fractional derivative. IJAAMM. 2(1) (2014) 83-91

[7] G. Adomian, Solving Frontier Problems of Physics: The Decomposition Method, Kluwer Academic, 1994.

[8] M.A. Abdou, A.A. Soliman, Variational iteration method for solving Burger's and coupled Burger's equations, Journal of Computational and Applied Mathematics 181 (2005) 245 - 251.

[9] Mountassir HamdiI Cherif, Kacem Belghaba And Djelloul Ziane, Homotopy Perturbation Method For Solving The Fractional Fisher's Equation, International Journal of Analysis and Applications, ISSN 2291-8639, Volume 10, Number 1 (2016), 9-16..

[10] Podlubny I. . Fractional differential equations. Academic press, New York; 1999

[11] J. Biazar, E. Babolian, R. Islam, Solution of the system of ordinary differential equations by Adomian decomposition method, Appl. Math. Comput. 147 (3) (2004) 713-719 Comput. 147 (3) (2004) 713-719.

[12] Ji-Huan He, Variational iteration method - a kind of non-linear analytical technique: some examples, International Journal of Non-Linear Mechanics 34 (1999) 699-708.

[13] S.G. Samko, A.A. Kilbas, O.I. Marichev, Fractional Integrals and Derivatives: Theory and Applications, Gordon and Breach, Yverdon, 1993

[14] Shaher Momani, Zaid Odibat, Homotopy perturbation method for nonlinear partial differential equations of fractional order, Physics Letters A, Volume 365, Issues 5-6, 11 June 2007, Pages 345-350.

[15] S. Gh. Hosseini, F. Mohammadi. A New Operational Matrix of Derivative for Chebyshev Wavelets and its Applications in Solving Ordinary Differential Equations with Non analytic Solution. AMS, 5 (51) (2011) 25372548.

[16] V. E. Tarasov, Fractional integro-di_erential equations for electromagnetic waves in dielectric media, Theoretical and Math. Phys, 158 (2009), 355-359.

[17] W. A. Khan, F. A. Ansari, European Option Pricing of Fractional Black-Scholes Model Using Sumudu Transform and its Derivatives, General Letters in Mathematics, Vol 1 (3) 2016 pp 74-80. 